\title{
Exogenous ocular candidiasis associated with intravenous heroin abuse
}

\author{
TANIA C. SORRELL, ${ }^{2}$ CATHERINE DUNLOP, ${ }^{3}$ PETER J. COLlIGNON, ' AND \\ JOHN A. HARDING ${ }^{3}$
}

From the ${ }^{1}$ Infectious Disease Medical Unit, Westmead Centre, ${ }^{2}$ Department of Medicine, University of Sydney, and the ${ }^{3}$ Ophthalmology Unit, Westmead Centre, Australia

SUMMARY Seven young men developed disseminated candidiasis within 10 days of a single episode of intravenous heroin abuse. Sequential development of eye and skin lesions was noted in all cases. The bone or costal cartilage was involved in five. Ocular manifestations of candidiasis included episcleritis, chorioretinitis, and endophthalmitis. A presumptive diagnosis of candida chorioretinitis was established rapidly by culture of Candida albicans from involved skin and costal cartilage. Systemic therapy with amphotericin B plus 5-fluorocytosine resulted in cure of the episcleritis, chorioretinitis, osteomyelitis, costochondritis, and skin infection. Pars plana vitrectomy with local instillation of amphotericin $B$ was required to cure chorioretinitis associated with vitreal extension of infection.

The importance of eye lesions in the clinical diagnosis of disseminated candidiasis and in determination of therapy has been appreciated only recently. ${ }^{12}$

In a review of ocular manifestations of candida septicaemia characteristic white, fluffy, chorioretinal lesions with or without haemorrhage or extension into the vitreous humour were described. Less common lesions included Roth spots, papillitis, iritis, abscesses of the ciliary body, inflammation of the anterior chamber, and conjunctivitis. Deep organ involvement was identified in $80 \%$ of patients with endophthalmitis. ${ }^{2}$

The incidence and evolution of eye and other metastatic lesions in candidiasis is poorly documented, as patients usually present with disseminated disease following an unidentified episode of fungaemia. In September 1982 we identified a limited outbreak of disseminated candidiasis, presumed to be due to intravenous inoculation of contaminated heroin. The purpose of this paper is to describe the development of ocular lesions in these patients and to discuss aspects of early diagnosis and optimal management.

\section{Materials and methods}

In September 1982 a 26-year-old male presented to Westmead Hospital with disseminated candidiasis Correspondence to Dr T. C. Sorrell, Department of Medicine, Westmead Centre, Westmead NSW 2145, Australia. following a single episode of intravenous abuse of heroin. Eight friends had also injected themselves with heroin from the same batch on the same night, and were contacted for follow-up. Two of the eight had boiled the heroin prior to injection and remained well. Disseminated candidiasis was diagnosed in the other six patients. Six of the seven patients with candidiasis were admitted to hospital. Clinical evidence of systemic infection was sought by daily general physical examination. Ocular examinations included visual acuity, slit-lamp examination, tonometry, direct, indirect and Goldmann threemirror funduscopy. Episcleritis was diagnosed in the presence of localised anterior subconjunctival and episcleral injection, with overlying chemosis and minimal local discomfort. Chorioretinitis was identified as white circumscribed retinal lesions with or without haemorrhage, or overlying vitreous haze. Endophthalmitis was diagnosed when retinal lesions extended anteriorly into the vitreous humour.

\section{Case reports}

CASE 1

The index case was a 26-year-old man who was admitted to hospital with fever, anorexia, and blurred vision. Ten days previously he and eight friends had injected themselves intravenously with heroin. Six hours after injection the patient experienced anorexia, then nausea and vomiting, generalised 
aches, and abdominal pain. The pain abated after six hours. The anorexia and nausea persisted. Three days after the injection he noted blurring of vision, occasional 'spots' before the eyes, and 'redness' of both eyes, especially the right. Jaundice was also noted on day 3; this increased over two days, then subsided. On the fifth day he first noticed 'pimples' in the scalp and beard area; these subsequently became more numerous and appeared in axillary and pubic hair-bearing areas. On the day of presentation (day 10) the patient complained of pain over the right eighth costal cartilage. He had been well in the past and had abused heroin twice in the 12 months before admission.

On physical examination his temperature was $37 \cdot 4^{\circ} \mathrm{C}$. Multiple pustules and subcutaneous nodules were associated with hair follicles in the scalp and beard area. A few pustules were present in the left axillary and pubic hair-bearing areas. Enlarged, nontender, occipital lymph nodes were present bilaterally. Cardiovascular examination was normal. A small area of tenderness and minimal swelling was found at the eighth costochondral junction on the right side. General examination was otherwise normal.

The eyes showed bilateral conjunctival injection. On ophthalmoscopy the right vitreous humour appeared hazy. The right macula was obscured by two rounded, white lesions which extended anteriorly into the vitreous humour. The optic disc appeared oedematous. In the left eye, two small areas of chorioretinitis were noted near the fovea. Slit-lamp examination revealed cells in the vitreous humour and anterior chamber of each eye. Visual acuity was $2 / 60$ for the right eye and $6 / 6$ for the left.

Investigations showed abnormal liver function with gamma glutamyl transpeptidase $255 \mathrm{U} / 1$ (normal 843), alkaline phosphatase $159 \mathrm{U} / \mathrm{l}$ (normal 30-115), bilirubin $15 \mu \mathrm{mol} / \mathrm{l}$ (normal 2-21). The creatinine was $73 \mu \mathrm{mol} / \mathrm{l}$ (normal 60-125), haemoglobin $13.0 \mathrm{~g} / \mathrm{dl}$, white cell count $16.3 \times 10^{\%} / 1$ with $70 \%$ neutrophils, platelets $328 \times 10^{9} / 1$, ESR $61 \mathrm{~mm} / \mathrm{h}$. The titre of antibody to Candida albicans (indirect haemagglutination) was 10240 (negative if less than or equal to 160), cytomegalovirus titre 4 , toxoplasmosis haemagglutination titre 256 , toxoplasmosis complement fixation titre less than 4 , serum cryptococcal antigen negative, hepatitis $B$ surface antibody positive. Chest $x$-ray was normal. Microbiological samples were obtained from pubic lesions, facial lesions, and neck and scalp biopsies. Candida albicans was isolated on blood agar and Sabouraud's medium within 48 hours in all cases. Forty-eight hour subcultures from Robertson's cooked meat medium also yielded Candida albicans. The urine contained a trace of protein and $10-1000 \times 10^{9}$ leucocytes $/ 1$. Three blood and urine cultures, including fungal cultures, were

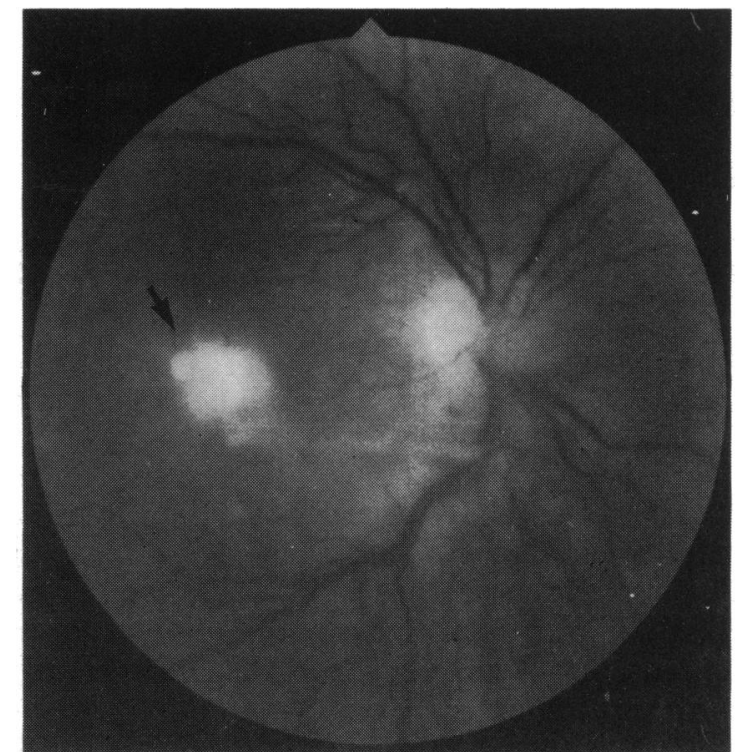

Fig. 1 Right eye of the index case immediately prior to initiation of antifungal therapy. A large fluffy exudate (arrow) with surrounding smaller lesions is seen in the macular area. A small haemorrhage is located inferiorly to the optic disc. The nasal margin of the optic disc is blurred.

negative. A ${ }^{99 \mathrm{~m}}$ technetium bone scan demonstrated an area of increased uptake at the medial end of the eighth right rib. Histology of a scalp biopsy showed acute folliculitis. Blastospores and pseudohyphae were present in a few of the most inflamed hair follicles. Pseudohyphae were also identified in hair shafts.

The patient was treated with oral 5-fluorocytosine (150 $\mathrm{mg} / \mathrm{kg} / \mathrm{day})$ and intravenous amphotericin B (maintenance $0.5 \mathrm{mg} / \mathrm{kg} /$ day). The skin lesions, cartilage pain, and hepatitis resolved after 20 days of therapy; the serum creatinine rose to $175 \mu \mathrm{mol} / \mathrm{l}$.

The areas of chorioretinitis in the left eye remained static for one week, then regressed. The lesions in the right eye progressed on therapy (Figs. 1,2). A right pars plana vitrectomy was performed seven days after institution of systemic antifungal therapy and amphotericin B, $5 \mu \mathrm{g}$, was instilled slowly, anterior to the retina. Material obtained at vitrectomy did not yield Candida albicans on culture of the concentrate, but Gram stain revealed budding yeasts. The postoperative course was complicated by intraocular haemorrhage and uveitis. Vision in the eye was restricted to appreciation of hand movements only. A course of $1016 \mathrm{mg}$ of amphotericin B with concomitant 5-fluorocytosine was completed over 31 days.

On review four months later the patient remained 


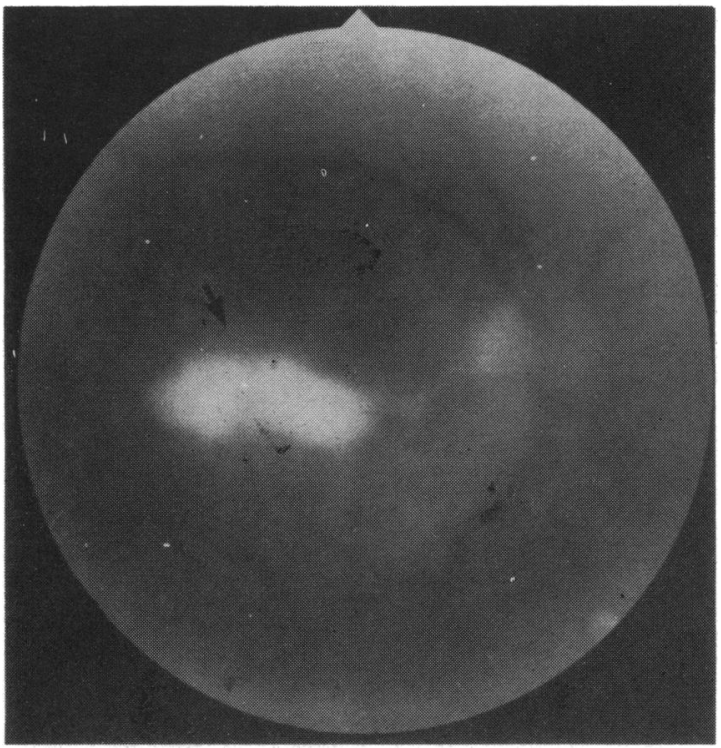

Fig. 2 Right eye of the index case on the seventh day of antifungal therapy. The fundus appears blurred due to generalised vitreal haze. The macular exudates have enlarged (arrow) and are extending anteriorly into the vitreous humour.

asymptomatic. General physical examination was normal. Two small residual areas of pigmentation were present in the left eye; visual acuity was normal. In the right eye central vision was reduced to appreciation of hand movements and peripheral vision to appreciation of objects. The right retina could not be visualised because of residual vitreous haemorrhage. Slit-lamp examination revealed clearance of cells from the anterior chamber. The serum antibody titre to Candida albicans had fallen to 640 .
CASES 2-7

The six other patients had injected themselves with heroin on the night of 26 August 1982. Two different syringes and two samples of heroin, obtained from the same dealer, were used. Juice from the same lemon was used as an additive. None of these materials was available for culture. Symptoms experienced by these patients were similar to those of the index case. Clinical findings are summarised in Table 1.

\section{OCULAR FINDINGS IN DISSEMINATED CANDIDIASIS \\ Episcleritis}

Of the 14 eyes examined episcleritis was present in three. Mild, local discomfort and redness of the eyes were noted one to two days after injection of heroin. Examination approximately 15 days later revealed no evidence of anterior or posterior uveitis. An area of unilateral episcleritis overlay the insertion of the left medial rectus muscle in one patient. Bilateral episcleritis in a second patient was confined to the area over the insertion of the inferior rectus muscles.

Histological examination of conjunctival biopsies one week after the start of antifungal therapy revealed mild, non-specific inflammation. Cultures and staining of sections with periodic acid Schiff reagent and Gomori methenamine silver reagent were negative for fungi.

\section{Chorioretinitis and endophthalmitis}

Six patients complained of blurred vision, 'floaters,' and redness of the eye(s) four to six days after injection of heroin. Chorioretinitis was noted on examination of five patients; bilateral involvement was seen in three of these. On slit-lamp examination of a further patient in whom there was no evidence of chorioretinitis a few cells were noted in the vitreous humour.

Table 1 Features of disseminated candidiasis following heroin abuse

\begin{tabular}{|c|c|c|c|c|c|c|c|c|}
\hline Patient & Chorioretinitis & $\begin{array}{l}\text { Vitreal } \\
\text { extension }\end{array}$ & Episcleritis & ${ }^{*}$ Hepatitis & $\begin{array}{l}\text { †Osteo- } \\
\text { myelitis }\end{array}$ & $\begin{array}{l}\text { Costo- } \\
\text { chondritis }\end{array}$ & $\begin{array}{l}\text { Skin } \\
\text { lesions }\end{array}$ & $\begin{array}{l}\text { Indirect } \\
\text { haemagglutination } \\
\text { to C. albicans at } \\
\text { presentation }\end{array}$ \\
\hline 1 & Bilateral & $+($ Unilateral) & - & + & + & + & + & 10240 \\
\hline 2 & Unilateral & - & + & + & - & + & + & 2560 \\
\hline 3 & Unilateral & - & - & + & + & - & + & 1280 \\
\hline 4 & Bilateral & - & - & + & + & + & + & 5120 \\
\hline 5 & Bilateral & $\ddagger$ (Unilateral) & - & + & - & - & + & 1280 \\
\hline 6 & Nil & - & + & + & - & + & + & 5120 \\
\hline 7 & Nil & $\begin{array}{l}\text { Cells in } \\
\text { vitreous } \\
\text { (bilateral) }\end{array}$ & - & + & - & - & + & 2560 \\
\hline
\end{tabular}

${ }^{*}$ Hepatitis defined by abnormal serum liver enzyme levels.

† Bone scan positive in each.

$¥$ This patient had received systemic prednisone $20 \mathrm{mg} / \mathrm{d}$ plus predsol eye drops, for 7 days prior to admission. 


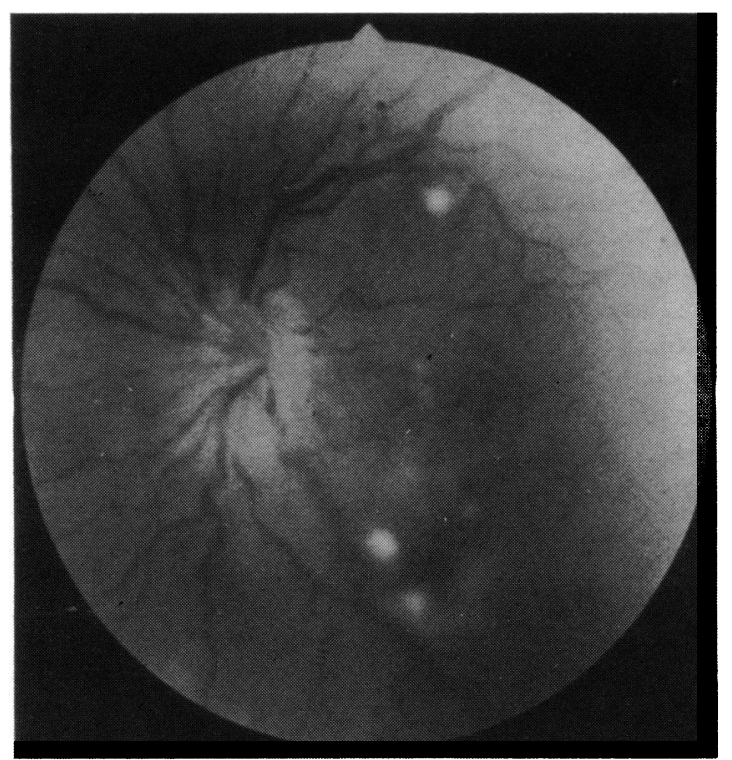

Fig. 3 Multiple fluffy exudates with vitreal extension are seen in the left fundus. Small haemorrhages are present peripherally. Generalised blurring of the optic disc is well demonstrated.

The foci of chorioretinitis were located at the posterior pole of the eye, usually near the macula. Lesions adjacent to the optic disc (two eyes) were associated with blurring of the disc margin (Figs. 1 and 3). In one case the lesion was adjacent to the cilioretinal artery. Coarse pigmentary changes were noted in four eyes. A single Roth spot was present in the inferior temporal quadrant of one eye (Fig. 4). The chorioretinal lesions varied in size from small 'dots' to large, pale, fluffy lesions which extended into the vitreous humour.

\section{LABORATORY DIAGNOSIS}

Candida albicans was cultured from skin lesions in all patients and from costal cartilage in one. Indirect haemagglutinating antibody titres were elevatec' in all (Table 1). Two patients underwent vitrectomy seven and 21 days respectively after commencement of antifungal therapy; Candida albicans was not cultured from surgical samples of vitrectomy fluid.

\section{MANAGEMENT}

Therapy with amphotericin B and 5-fluorocytosine was instituted in six of the seven patients. Four patients received approximately $1 \mathrm{~g}$ of amphotericin B. Skin, musculoskeletal, and small eye lesions were cured by this therapy. A therapeutic pars plana vitrectomy with local instillation amphotericin B (5 $\mu \mathrm{g})$ was undertaken in two patients after progression of chorioretinal lesions on systemic antifungal

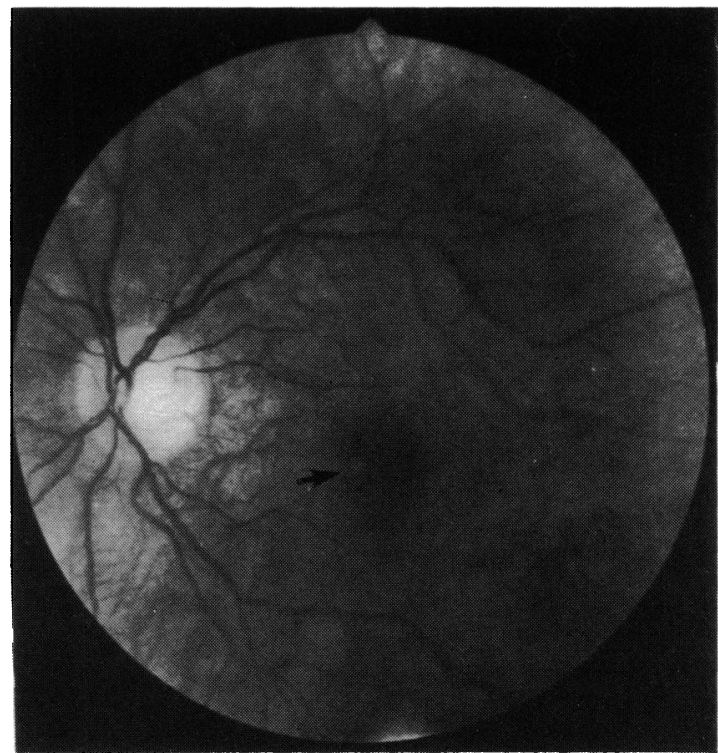

Fig. 4 A single Roth spot (arrow) is seen clearly in the paramacular area of the fundus of the left eye.

therapy. This procedure, although curative of the infection, was complicated by intravitreal haemorrhage and persistence of poor visual acuity in both cases. A further patient, (patient 2, Table 1), who absconded after receiving $300 \mathrm{mg}$ of amphotericin $B$, returned five weeks later with increased pain and swelling over his sixth left costal cartilage and again refused treatment. The small eye lesions seen initially had resolved. He was then lost to follow-up. The seventh patient presented with mild folliculitis. Eye examination revealed only occasional cells in the vitreous humour. These lesions resolved spontaneously.

\section{Discussion}

Characteristic chorioretinal and other eye lesions are an important clinical indicator of disseminated candidiasis. ${ }^{2}$ Certain eye lesions may be peculiar to heroin abusers-for example, vitreous involvement without chorioretinitis as observed by us and Snip et $a .^{3}$ and prominent inflammation of the anterior chamber. ${ }^{4}$ Episcleritis, present in two of our cases, has not been described previously. As Candida albicans was not isolated from conjunctival biopsies obtained from these patients after one week of anti:fungal therapy, evidence for the aetiological role of this agent in the episcleritis remains circumstantial.

Macronodular or follicular skin lesions confined to hair-bearing areas, and associated with occipital lymphadenopathy, were distinctive clinical features of our cases and may be characteristic of disseminated 
candidiasis in heroin abusers. ${ }^{5}$ The diagnosis of candidiasis was made readily by histological examination and culture of material from these skin lesions and supported by high titres of serum haemagglutinating antibody to Candida albicans. In one patient $C$. albicans was also isolated from an area of costochondritis. Culture of samples obtained from two patients at vitrectomy were negative for $C$. albicans, despite concentration of the specimens. Other workers have reported improvement in yield of fungal organisms after concentration of samples. ${ }^{4}$ Previous antifungal therapy may have affected yields from our patients. Serological tests have often been unhelpful in the diagnosis of disseminated candidiasis, particularly in immunosuppressed patient.' ${ }^{\prime}$ High titres were found at presentation in our cases, all previously normal hosts; titres were substantially lower at the end of therapy, consistent with recent candidal infection.

Optimal regimens of antifungal therapy for endophthalmitis, chorioretinitis, and disseminated candidiasis have not been defined. Small lesions may resolve spontaneously (our case 7). Courses of up to $2 \mathrm{~g}$ of amphotericin $\mathrm{B}$ have been recommended for deep-seated infection.' Combinations of amphotericin B and 5-fluorocytosine have been recommended because of potential synergistic activity against Candida albicans and prevention of the emergence of resistance to 5-fluorocytosine by the use of amphotericin B. ${ }^{\prime}$ Cure was achieved with $1 \mathrm{~g}$ of amphotericin B plus 5-fluorocytosine $(150 \mathrm{mg} / \mathrm{kg} /$ day $)$ in four of our cases. However, progression of eye lesions occurred during systemic therapy in two patients with marked endophthalmitis. In one case administration of systemic corticosteroids prior to diagnosis may have promoted intravitreal extension of chorioretinitis. Therapeutic vitrectomy plus intravitreal instillation of amphotericin $B$ were curative in both patients. This has been the experience of other workers. ${ }^{46}$ The value of intravitreal instillation of amphotericin B remains controversial owing to its potential retinal toxicity ${ }^{6-8}$ and the difficulty of assessing its efficacy. Cure of endophthalmitis has been achieved by vitrectomy in association with systemic antifungal therapy, ${ }^{39}$ and intravitreal amphotericin B has been used without resultant retinal toxicity. ${ }^{111}$ However, pooling of high concentrations of antifungal drugs in unformed vitreous may result in local toxicity. ${ }^{12}$ Uveitis and retinal and preretinal haemorrhages were noted after local instillation of amphotericin B in our two patients who underwent this procedure; these complications may have been caused by local drug toxicity.
The need for surgery to cure vitreal candidiasis reflects in part the poor penetration of amphotericin $B$ into the vitreous humour. ${ }^{13}$ Candidal infection confined to the choroid and retina may be cured by systemic therapy alone, as noted by ourselves and others. ${ }^{2} 14$ is

Further studies are required to determine the role of amphotericin B and less toxic drugs such as ketoconazole in the management of ocular candidiasis. Cases of failure of ketoconazole therapy in candida endophthalmitis have been reported ${ }^{16}$ suggesting that amphotericin $B$ will remain the mainstay of treatment.

We wish to thank Mr J. Gardiner and Dr R. Munro for microbiological assistance and Robyn Eggington for typing the manuscript.

\section{References}

1 Edwards JE, Lehrer RI, Stiehm ER, Fisher TJ, Young LS. Severe candidal infections. Clinical perspective, immune defense mechanisms and current concepts of therapy. Ann Intern Med; 1978; 89: 91-106.

2 Edwards JE Jr, Foos RY, Montgomerie JZ, Guze LB. Ocular manifestations of candida septicaemia: Review of seventy-six cases of hematogenous candida endophthalmitis. Medicine 1974; 53: 47-75.

3 Snip RC, Michels RG. Pars plana vitrectomy in the management of endogenous candida endophthalmitis. Am NOphthalmol 1976; 82: 699-704.

4 Aguilar GL, Blumenkrantz MS, Egbert PR, McCulley JP. Candida endophthalmitis after intravenous drug abuse. Arch Ophthalmol 1979; 97: 96-100.

5 Collignon PJ, Sorrell TC. Disseminated candidiasis. Evidence of distinctive syndrome in heroin abusers. $\mathrm{Br}$ Med $J$ 1983; 287: 861-2.

6 Tarr K. Candida endophthalmitis and drug abuse. Aust J Ophthalmol 1980; 8: 303-5.

7 Souri EN, Green WR. Intravitreal amphotericin B toxicity. Am J Ophthalmol 1974; 78: 77-81.

8 Axelrod AJ, Peyman GA, Apple DJ. Toxicity of intravitreal injection of amphotericin B. Am J Ophthalmol 1973; 76: 578-83.

9 Billson FA, Playfair TJ. The place for vitrectomy in posterior segment infection. Aust J Ophthalmol 1981; 9: 21-5.

10 Stern GA, Fetkenhour CL, O'Grady RB. Intravitreal amphotericin B treatment of candida endophthalmitis. Arch Ophthalmol 1977; 95: 89-93.

11 Perraut LE Jr, Perraut LE, Bleiman B, Lyons J. Successful treatment of Candida albicans endophthalmitis with intravitreal amphotericin B. Arch Ophthalmol 1981; 99: 1565-7.

12 Tolentino FI, Foster S, Lahav M, Liu LHS, Rabin AR. Toxicity of intravitreous miconazole. Arch Ophthalmol 1982; 100: 1504-9.

13 Weinstein L. Miscellaneous antibacterial agents: antifungal and antiviral agents. In: Goodman LS, Gilman A, eds. The pharmacological basis of therapeutics. New York: Macmillan, 1970: 1237.

14 Horne MJ, Ma MH, Taylor RF, Williams R, Zylstra W. Candida endophthalmitis. Med J Aust 1975; 1: 170-2.

15 Ho PC, O'Day DM. Candida endophthalmitis and infection of costal cartilages. BrJ Ophthalmol 1981; 65: 333-4.

16 Drouhet E, Dupont B. Laboratory and clinical assessment of ketoconazole in deep-seated mycoses. AmJ Med 1983; 74:30-47. 